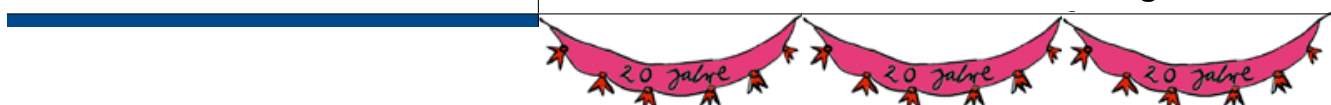

Nature bezeichneten das Geschehen 2008 als unglaublichen Schwindel, doch viele haben es geglaubt!

Eine traurige Geschichte ist mit der weiten Verbreitung von Nitinol-Käfigen verbunden, die zeitweise in strikturierte Harnröhren eingesetzt wurden. Leider wucherte das Urothel durch die Maschen hindurch und machte eine Entfernung notwendig, bei der dann meist die gesamte Harnröhre mit entfernt wurde.

Die letzten zwei Jahrzehnte in der Urologie waren aber auch eine prospe- rierende Zeit mit vielen Innovationen, die häufig aus Deutschland kamen: Die Neoblase ist heute Standard und revolutionierte die Harnableitung, gleiches gilt für den Pouch. Die extrakorporale Stoßwellenlithotripsie (ESWL) hat die Steintherapie nachhaltig verändert und wird heute sicher unterschätzt. Das TVT („Tension free Vaginal Tape“) hat eine neue Art der Behandlung der Stressinkontinenz eingeleitet, wobei ich glaube, dass bis heute keiner so ganz genau weiß, warum es oftmals funktioniert. Mit der sogenannten Schwellkörperautoinjektionstechnik (SKAT) entstand ein neuer Fachzweig in der Urologie und wer hätte 1985 gedacht, dass einmal ein Herzmittel als blaues Wunder die Welt der Erektionsschwäche und des Alters nachhaltig verändern würde. Es gab hoffnungsvolle Innovationen, die bis heute ein Erfolg sind, doch gab es auch Fehlentwicklungen und Überschätzungen oft aus übertriebenem Ehrgeiz -, aus denen man lernen sollte.

Prof. Dr. med. Thomas-Alexander Vögeli

\title{
Tops und Flops in 20 Jahren Urologie
}

$Z_{\mathrm{in}}^{\mathrm{u}}$ u den Tops der vergangenen 20 Jahre in der Urologie gehört der Durchbruch der Aktiven Überwachung (AS) in der Behandlung des lokal begrenzten Prostatakarzinoms (PCa) der Low-RiskGruppe. Epidemiologische Daten, Kohortenstudien, Ergebnisse der Versorgungsforschung und Krebsregisterdaten zeigen, dass nicht nur randomisierte Studien zu einem neuen Standard führen. Inzwischen bestätigen drei systematische Reviews, dass unsere Entscheidung, AS in die 2014 aktualisierte Fassung der S3-Leitlinie aufzunehmen - trotz damaliger Widerstände - richtig war. Die ASGegner hatten in der Leitlinienkommission mit den guten Ergebnissen der radikalen Prostatektomie (RP) argumentiert. Als Beweis für deren Überlegenheit benutzten sie Ergebnisse der randomisierten Studie von Bill-Axelson (2013). Diese Studie muss aber richtig gelesen werden: Obwohl man dort lokal fortgeschrittene Tumoren mit positivem Tast- befund und PSA-Werten $\leq 50 \mathrm{ng} / \mathrm{ml}$ einbezogen hatte, waren die Unterschiede zwischen Operation und Watchful Waiting (WW) gering und auf < 65-Jährige beschränkt. Die PIVOT-Studie bestätigte diese Ergebnisse. Dass AS nicht mit WW, einer palliativen Behandlung, gleichgesetzt werden darf, wurde erst allmählich begriffen - obwohl dies seit 2006 bekannt ist.

Der absolute Flop der letzten beiden zwei Jahrzehnte ist PREFERE, „die weltweit größte klinische Studie zum lokal begrenzten Prostatakarzinom" (Dtsch. Ärztebl. vom 23. Januar 2013). Unterstützt von Fachgesellschaften, dem Berufsverband Deutscher Urologen, der Prostatakrebsselbsthilfe und Persönlichkeiten maßgeblicher Institutionen warb man mit dem Argument, man wisse nicht, welche Option - RP, perkutane Bestrahlung, Brachytherapie, AS - die beste sei. Dieses Problem wäre nur mit der Studie zu lösen. Sachliche Kritik an

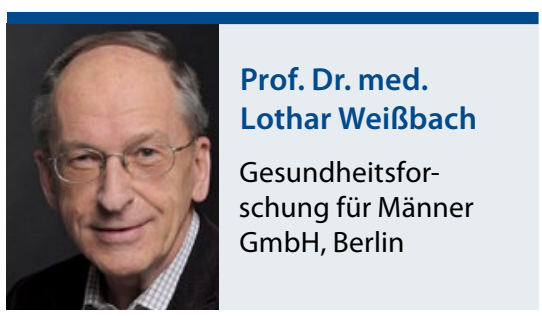

der Planung, der großen Zahl benötigter Patienten sowie der langen Laufzeit wurde ignoriert. Stattdessen wurde, wie es Kritiker der Studie auf einer Pressekonferenz in München am 2.11.2016 deklarierten, aus der „Meilenstein-Studie“ ein „25-Millionen-Desaster“. Die Fehlannahme einer $10 \%$-Mortalität, ein schwieriges Studiendesign und ethische Bedenken wegen der Übertherapie in drei Armen führten zu mangelhafter Rekrutierung und endlich zum Abbruch der Studie. Nun ist es an der Zeit, aus den planungsbedingten Fehlern der Studie zu lernen, die hierzulande verbreitete Übertherapie aufzugeben und durch ein risikoadaptiertes Vorgehen zu ersetzen.

Prof. Dr. med. Lothar Weißbach

\section{Die größten (Miss-)Erfolge in der Urologie}

\begin{abstract}
$\Delta$ ls eine große Errungenschaft der letzten Jahre empfinde ich die Etablierung von Leitlinien (Top!) und damit auch die multidisziplinäre Vorgehensweise. Nachteilig ist der damit verbundene große Zeitaufwand, der meist unzureichend honoriert wird. Auch hinsichtlich verschiedener Krankheitsbilder
\end{abstract}

in der Urologie finden sich zahlreiche Tops, aber auch Flops.

Zu den Tops bei der obstruktiven Prostatavergrößerung zählen Laserverfahren, die bipolare Resektionstechnik (TURiSTechnik), Enukleation und Alphablocker. Als Flop offenbarten sich Stents für die prostatische Harnröhre. Beim Prostata-

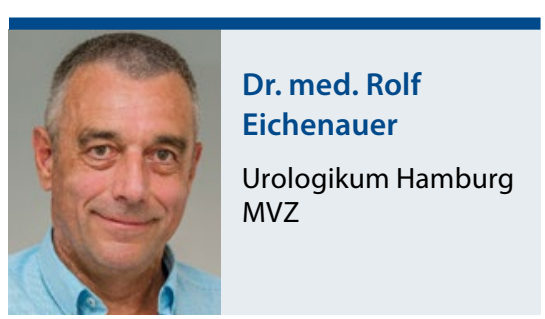

karzinom ist eine Vielzahl an Tops zu benennen, unter anderem die Entwicklung 